\title{
Selective inhibitory activity against MAO and molecular modeling studies of 2-thiazolylhydrazone derivatives.
}

Franco Chimenti, Elias Maccioni, Daniela Secci ${ }^{*}$, Adriana Bolasco, Paola Chimenti, Arianna

Granese, Olivia Befani, Paola Turini, Stefano Alcaro, Francesco Ortuso, Maria C. Cardia, Simona

Distinto.

Contents

Title page

S1

Chemistry

S2 


\section{Chemistry}

Melting points are uncorrected and were determined on a Reichert Kofler thermopan apparatus. ${ }^{1} \mathrm{H}-$ NMR spectra were recorded on a Bruker AMX (300 MHz) using tetramethylsilane (TMS) as internal standard and DMSO or $\mathrm{CDCl}_{3}$ as solvents (chemical shifts in $\delta$ values, $J$ in $\mathrm{Hz}$ ). Electron ionisation (EI) mass spectra were obtained by a Fisons QMD 1000 mass spectrometer $(70 \mathrm{eV}, 200 \mu \mathrm{A}$, ion source temperature $200^{\circ} \mathrm{C}$ ). The samples were introduced directly into the ion source. Elemental analyses for $\mathrm{C}, \mathrm{H}$, and $\mathrm{N}$ were performed on a Perkin-Elmer $240 \mathrm{~B}$ microanalyser, and the analytical results were within $\pm 0.4 \%$ of the theoretical values.

Table 1 Chemical physical properties of derivatives 15 and $\mathbf{1 6 .}$

\begin{tabular}{|c|c|c|c|c|c|c|}
\hline Comp & $\mathbf{R}$ & $\mathbf{R}^{1}$ & $\begin{array}{c}\mathbf{M p} \\
{ }^{\circ} \mathrm{C}\end{array}$ & $\begin{array}{c}\text { Yield } \\
\%\end{array}$ & Formula & ${ }^{1}$ H-NMR \\
\hline 15 & & $\mathrm{CH}_{3}$ & $185-7$ & 59 & $\mathrm{C}_{12} \mathrm{H}_{14} \mathrm{ClN}_{3} \mathrm{~S}$ & $\begin{array}{l}{ }^{1} \mathrm{H}-\mathrm{NMR}\left(\mathrm{CDCl}_{3}\right): \delta 2.41\left(\mathrm{~s}, 3 \mathrm{H}, \mathrm{CH}_{3}\right) ; \\
2.52\left(\mathrm{~s}, 3 \mathrm{H}, \mathrm{CH}_{3}\right) ; 6.24\left(\mathrm{~s}, 1 \mathrm{H}, \mathrm{C}_{5} \mathrm{H}-\right. \\
\text { thiaz. }) ; .25\left(\mathrm{t}, 2 \mathrm{H}, 2-\mathrm{CH}_{3} \text {-phenyl, Jo } \mathrm{Hz}=\right. \\
\text { 7.6); } 7.33\left(\mathrm{dd}, 1 \mathrm{H}, 2-\mathrm{CH}_{3}-\text { phenyl, Jo } \mathrm{Hz}=\right. \\
7.1, \mathrm{Jm} \mathrm{Hz}=1.3) ; 7.76\left(\mathrm{~d}, 1 \mathrm{H}, 2-\mathrm{CH}_{3}-\right. \\
\text { phenyl, } \mathrm{Jo} \mathrm{Hz}=7.6) ; 8.66(\mathrm{~s}, 1 \mathrm{H},=\mathrm{CH}- \\
\text { phenyl); } 13.30\left(\mathrm{~s}, 1 \mathrm{H}, \mathrm{NH}, \mathrm{D}_{2} \mathrm{O} \text { exch). }\right.\end{array}$ \\
\hline 16 & & $\mathrm{C}_{6} \mathrm{H}_{5}$ & $220-1$ & 74 & $\mathrm{C}_{17} \mathrm{H}_{16} \mathrm{BrN}_{3} \mathrm{~S}$ & 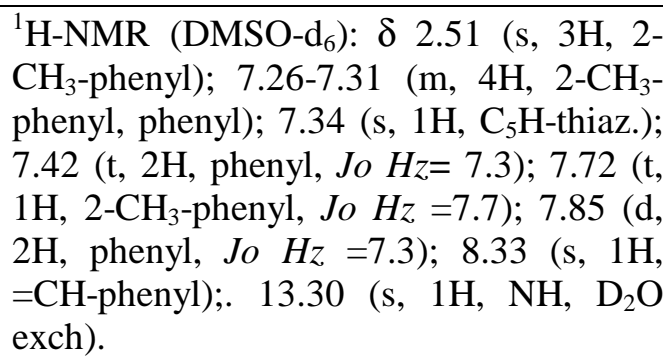 \\
\hline
\end{tabular}

